\title{
Microbiological Profile and Antibiotic Susceptibility Pattern of Urinary Tract Infections at Muthoot Medical Centre, Pathanamthitta, India
}

\author{
T. U. Jisha ${ }^{1}$ and Sara Mathew ${ }^{2 *}$ \\ ${ }^{1}$ Department of Microbiology, Mount Zion Medical college, Chayalode, Adoor, Kerala, India \\ ${ }^{2}$ Department of Microbiology, Muthoot Medical Centre, Pathanamthitta, Kerala, India \\ *Corresponding author
}

\begin{tabular}{l} 
Key w o r d s \\
Antibiotic policies, \\
Antibiotic \\
susceptibility, \\
Rational choice, \\
Urinary tract \\
infections \\
\hline Article Info \\
\hline Accepted: \\
25 March 2021 \\
Available Online: \\
10 April 2021 \\
\hline
\end{tabular}

\section{A B S T R A C T}

Urinary tract infections are one of the major health problem affecting both sexes of all age group. UTIs are often treated with different broad-spectrum antibiotics. Poor patient compliance and incomplete course of antibiotic therapy have resulted in the evolution of resistance to many of these antibiotics. The aim of this study was to determine the prevalence of bacteria causing urinary tract infections and their susceptibility pattern of patients reporting in MGM Muthoot Medical Centre Pathanamthitta. Mid stream urine (MSU) specimens sent to the laboratory from October 2018 to March 2019 were collected and inoculated onto blood agar and MacConkey agar and incubated at $37^{\circ} \mathrm{C}$ for 24 hours. Identification and antibiotic susceptibility test were done following standard operative procedures. 35\% (757/2179) samples showed a significant growth out of which 50\% (379/757) were females, 31\% (234/757) were males and 19\% (144/757) were children. Uropathogenic E. coli (UPEC) has been found to be the major pathogen causing UTI in all category of patients which account for 70\% (526/757) followed by Klebsiella spp. 19\% (140/757), Enterococcus spp. 6\% (38), Pseudomonas spp. 4\% (32), Proteus spp. 2\% (11) and Staphylococcus aureus 1\% (9).The higher rate of sensitivity among UPEC isolates were towards imipenem /meropenem (99\%), piperacillin - tazobactam (96\%), cefaperazone- sulbactam (96\%), amikacin (96\%) nitrofurantoin $(92 \%)$ and gentamicin (84 $\%)$. The most sensitive oral antibiotics were nitrofurantoin $(92 \%)$ followed by trimethoprim/sulfamethoxazole ie cotrimoxazole (61\%) and the quinolone group (43\%). These data may be used to determine trends in antimicrobial susceptibilities, to formulate local antibiotic policies in order to assist clinicians in the rational choice of antibiotic therapy to prevent misuse or overuse of antibiotics.

\section{Introduction}

Urinary tract infections are one of the major health problem affecting both sexes of all age group (Maleki et al., 2017). It is more common in females as compared to males due to shorter urethra \& sexually active life during reproductive age group (Humayun et al.,
2012). The other predisposing factors are pregnancy, use of contraceptive method, urinary tract abnormality, blockage in the tube due to kidney stones or benign prostatic hyperplasia, suppressed immune system or any other surgical intervention of the urinary tract (Tuli et al., 2016). 
Escherichia coli is the most widely recognized Gram-negative extra intestinal pathogen isolated from urine culture in patients with UTIs, whether complicated or uncomplicated, accounting for $70-80 \%$ of community acquired infections and 40- $60 \%$ of healthcare- associated infections (Hisano et al., 2015). Uropathogenic Escherichia coli (UPEC) utilize a variety of virulence factors such as surface structural components, like lipopolysaccharide, capsule, flagella, pili, outer membrane proteins, as well as secreted toxins and siderophore.

Consequently, these mechanisms are double edged weapon as they play an important role in UTI pathogenesis furthermore; they are attractive candidates for the development of new drugs and vaccines (Werneburg et al., 2015 \& O'Brien et al., 2016).

Urinary tract infections, caused by multidrug resistant UPEC are alarmingly escalating. Widespread utilization of empirical antibiotics without performing anti-microbial susceptibility testing led to expanding resistance to different classes of antimicrobial agents generally prescribed among UPEC isolates in our country. In last decade bacteria emerged with new forms of virulence and new patterns of resistance to antimicrobial agents. World wide, Antimicrobial resistance among UPEC is a major health concern because of its expanding resistance to different classes of antimicrobial agents generally prescribed (Polse et al., 2016).

The increasing resistance to several broadspectrum antibiotics, utilized in the treatment of UTIs, for example (ampicillin, trimethoprim/sulfamethoxazole, fluoroquinolons, and cephalosporin), among clinical UPEC strains complicates treatment, increases costs, and decreases the effectiveness of the available antibiotics against this infection (Biedenbach et al., 2016). Hospitalized patients have a tendency to acquire UPEC infections with higher antimicrobial resistance (Lavigne et al., 2016). The proper selection of empirical antimicrobials and the effectiveness of antibiotic treatment necessitate a good understanding of the typical bacteriology involved in UTIs as well as the local resistance patterns, and the specific patient's antimicrobial and microbiologic history (Bartoletti et al., 2016). The estimation of local aetiology and susceptibility profile could support the most effective empirical treatment.

The aim of this study was to determine bacterial etiologic agent of uropathogen and evaluate their in vitro susceptibility pattern to commonly used antimicrobial agents.

\section{Materials and Methods}

\section{Study Design}

The study was conducted in the Department of Microbiology, MGM Muthoot Medical Centre Pathanamthitta from October 2018 to March 2019. The study included all the patients who were admitted or visited the outpatient department in the hospital with symptoms of UTI during the study period and had UTI confirmed by positive urine culture reports.

\section{Sample Collection and Processing}

Clean catch mid-stream urine was collected from patients referred by clinicians, insterile wide mouthed container labeled with in formation of patient's age, sex \& brief clinical history.

Proper instructions were given to the patients regarding the method of collection. In case of catheterized patients, catheter tube was cleaned \& clamped \& sample was collected a septically by disposable syringe. The sample was immediately transferred to microbiology laboratory for culture \& antibiotic sensitivity test. Urine sample was processed with in 2 hours of receiving $\&$ collection. 


\section{Direct Microscopy}

Wet mount examination was performed to look for the presence of pus cells, epithelial cells, crystals \& microorganisms.

\section{Bacterial Identification}

Urine sample was processed on MacConkey agar \& Blood agar by semi quantitative method with the help of sterile calibrated loop and incubated aerobically at 37 degree Celsius for about 24-48 hrs. Urine culture plates were examined for growth of bacteria. Growth of $>10^{5}$ colony forming unit $/ \mathrm{ml}$ was considered as significant. Identification of the bacteria was done on the basis of cultural characteristics, gram staining \& biochemical reactions.

The isolates were subjected to Antibiotics usceptibility teston Mueller Hinton agar by Kirby Bauer disk diffusion method as per CLSI(2017) guidelines using the commercially available antibiotic discs. In this study 11 groups of antibiotics were used which were quinolones, cephalosporins, aminoglycosides, aminopenicillins, carbapenems, sulphonamides, nitrofurantoin, fosfomycin, cindamycin, linezolid \&vancomycin

Lawn culture was performed on Mueller Hinton agar with the help of sterile cotton swab \& antibiotic discs were placed on the plate $\&$ incubated at 37 degree Celsius over night. Diameter of zone of inhibition is measured in millimetres as per guidelines provided by the manufacturer \& grouped into Sensitive, Intermediate \& Resistant.

\section{Results and Discussion}

A total of 2179 samples were collected in the study, of that 757 samples showed significant growth with culture positivity rate of $35 \%$. The remaining were either sterile or did not show any significant growth. Our result more or less correlates with the study of (Haque et al., 2015) who found that, the prevalence of UTIs was $46.67 \%$. In comparison to our result; (Pratap et al., 2016) found that prevalence rate of UTI was $32.5 \%$.

Among 757 positive samples 50\% (379/757) were females, $31 \%$ (234/757) were males and $19 \%(144 / 757)$ were children. This result is in accordance with other studies that females have a higher frequency of UTI compared to males (Salunke and Gidamudi, 2017; Mangal et al., 2017) reported similar finding. Similar results were shown by the Mahajan et al., that is due to the close proximity of the female urethra to the source of bacteria (eg. Anus, vagina) (Mahajan et al., 2016). Females are more prone to UTI than males as they lack bacteriostatic property of prostatic secretions (Al-Jebouri, 1989). This result is in accordance with other studies showed that females have a higher frequency of UTI compared to males (Salunke and Gidamudi, 2017; Mangal et al., 2017) reported similar finding. The anatomical site of the urogenital tract in females is in charge of bacterial contaminations caused by both internal and external flora (Shanthi and Kayathri, 2012).

E. coli has been found to be the major pathogen causing UTI in all category of patients which account for $70 \%$ (526/757) followed by Klebsiella spp. 19\% (140/757), Enterococcus spp.6\% (38), Pseudomonas spp. 4\% (32) Proteus spp. 2\% (11), and Staphylococcus aureus $1 \%$. This finding was in agreement with previous studies study done by (Stefaniuk et al., 2016; Kumar et al., 2016) in which similar results were reported. This result is in agreement with the previous studies of (Salunke and Gidamudi, 2017; Sanjee et al., 2017; Vranic et al., 2017; Wong et al., 2017) in which similar organisms were isolated, with E.coli was the most prevalent isolate.

The highest rate of isolation of $E$. coli causing 
UTI may be attributed to most of the bacterial organisms causing UTI originate from the faecal flora and among these facultative anaerobes, E. coli constitutes the major portion superimposed by various virulence factors that facilitate the ascent of bacteria from faecal flora, up the urethra into the bladder. They have several factors responsible for their attachment to uroepithelium such as adhesins, pili and fimbriae (Das et al., 2006).

The higher rate of sensitivity among UPEC isolates were towards imipenem /meropenem (99\%), piperacillin - tazobactam (96\%), cefaperazone- sulbactam (96\%), amikacin $(96 \%)$ nitrofurantoin $(92 \%)$ and gentamicin (84 \%). The most sensitive oral antibiotics were nitrofurantoin (92\%) followed by trimethoprim/sulfamethoxazole ie cotrimoxazole $(61 \%)$ and the quinolone group (43\%). Most of the isolates were resistant to ampicilin (92\% resistant). Cephalosporin group of antibiotics irrespective of their generation showed a sensitivity percentage of $31-33 \%$ except for ceftazidime which showed a higher sensitivity percentage (58\%).

This finding is in agreement with the study of
(Thangavel et al., 2017) who reported that the higher rate of sensitivity among UPEC isolate were towards Amikacin (96.3\%), Imipenem (96.3\%), and Nitrofurantoin $(89.7 \%)$. The susceptibility patterns of E.coli against different antibiotics vary in different geographical regions, eventually leading to empirical therapy which is based on the local susceptibility profiles. Bacterial biofilm are often associated with long-term persistence of organism in various environments. Bacteria in biofilm display dramatically increased resistance to antibiotics (Tajbakhsh et al., 2016).

Urinary tract infection (UTI) is one of the most frequent causes of nosocomial infections. Effective treatment of patients with UTIs commonly relies on the identification of the type of organisms and the selection of an effective antibiotic agent to the organism. The pattern of antimicrobial resistance of bacteria producing UTI varies in different regions. Monitoring of antimicrobial susceptibility can aid clinicians for prescribing appropriate antibiotics and in prevention of development of drug resistance.

Table.1 Isolated Pathogens With Their Prevalence Rate

\begin{tabular}{|c|c|c|c|}
\hline SI No. & Pathogen Isolated & $\begin{array}{c}\text { Total } \\
\text { No. }\end{array}$ & Percentage \\
\hline $\mathbf{1}$ & Escherichia coli & 526 & $\mathbf{7 0 \%}$ \\
\hline $\mathbf{2}$ & Klebsiella spp. & 140 & $\mathbf{1 9 \%}$ \\
\hline $\mathbf{3}$ & Enterococci spp & 38 & $\mathbf{6 \%}$ \\
\hline $\mathbf{4}$ & Pseudomonas spp & 32 & $\mathbf{4 \%}$ \\
\hline $\mathbf{5}$ & Proteus spp & 11 & $\mathbf{2 \%}$ \\
\hline $\mathbf{6}$ & Staphylococcus aureus & $\mathbf{9}$ & $\mathbf{1 \%}$ \\
\hline
\end{tabular}


Table.2 Antibiotic Discs Used

\begin{tabular}{|c|c|c|}
\hline Antibiotics & $\boldsymbol{\mu g}$ & Symbol \\
\hline Ampicillin & 10 & AMP \\
\hline Amoxy-clav & 30 & AMC \\
\hline Cephalexin & 30 & CN \\
\hline Cefuroxime & 30 & CXM \\
\hline Cefixime & 5 & CFM \\
\hline Cefotaxime/ceftriaxone & 30 & CTX/CTR \\
\hline Ceftazidime & 30 & CAZ \\
\hline Cefipime & 30 & CPM \\
\hline Ciprofloxacin & 5 & CIP \\
\hline Norfloxacin & 10 & NX \\
\hline Ofloxacin & 5 & OF \\
\hline Gentamicin & 10 & GEN \\
\hline Amikacin & 30 & AK \\
\hline Co-trimoxazole & 25 & COT \\
\hline Nitrofurantoin & 300 & NIT \\
\hline Imipenem/ meropenem & 10 & IMP/MRP \\
\hline Piperacillin- tazobactam & $100 / 10$ & PIT \\
\hline Cefaperazone- sulbactam & $75 / 30$ & CFS \\
\hline Colistin & 10 & CL \\
\hline Penicillin & $10 U$ & P \\
\hline Clindamycin & 2 & CD \\
\hline Fosfomycin & 200 & FO \\
\hline Linezolid & 30 & LZ \\
\hline High level gentamicin & 120 & HLG \\
\hline Vancomycin & 30 & VA \\
\hline Tigecycline & 15 & TGC \\
\hline
\end{tabular}


Int.J.Curr.Microbiol.App.Sci (2021) 10(04): 903-913

Table.3 Antibiotic Sensitivity Pattern Of Commonly Isolated Uropathogens

\begin{tabular}{|c|c|c|c|c|c|c|c|c|c|c|c|c|c|c|c|c|c|c|c|c|c|c|c|}
\hline ORGANISM & AMP & AMC & $\mathbf{C N}$ & CXM & CFM & CTX & $\mathrm{CAZ}$ & CPM & CIP & NX & OF & GEN & AK & COT & NIT & IMP & PIT & $\begin{array}{c}\text { CF } \\
\text { S }\end{array}$ & $\mathbf{P}$ & CD & FO & $\mathbf{L Z}$ & VA \\
\hline E.COLI & 8 & 31 & 32 & 32 & 33 & 33 & 58 & 38 & 42 & 43 & 44 & 84 & 96 & 61 & 92 & 99 & 96 & 96 & - & - & - & - & - \\
\hline KLEBSIELLA & $\mathbf{0}$ & 25 & 20 & 38 & 37 & 37 & 46 & 48 & 57 & 58 & 61 & 79 & 77 & 67 & 65 & 84 & 74 & 74 & & & & & \\
\hline PSEUDOMONAS & - & - & - & - & - & - & 50 & 43 & 43 & 39 & 43 & 54 & 64 & - & - & 64 & 64 & - & - & - & - & - & - \\
\hline ENTEROCOCCUS & 64 & - & - & - & - & - & - & - & 17 & 17 & 18 & 67 & - & - & 89 & - & - & - & 8 & - & 70 & 100 & 100 \\
\hline STAPHYLOCOCCUS & - & - & - & - & - & - & & - & 22 & - & - & 81 & 85 & 72 & 93 & - & - & - & 21 & 71 & - & 100 & 100 \\
\hline
\end{tabular}


Fig.1 Percentage of UTI

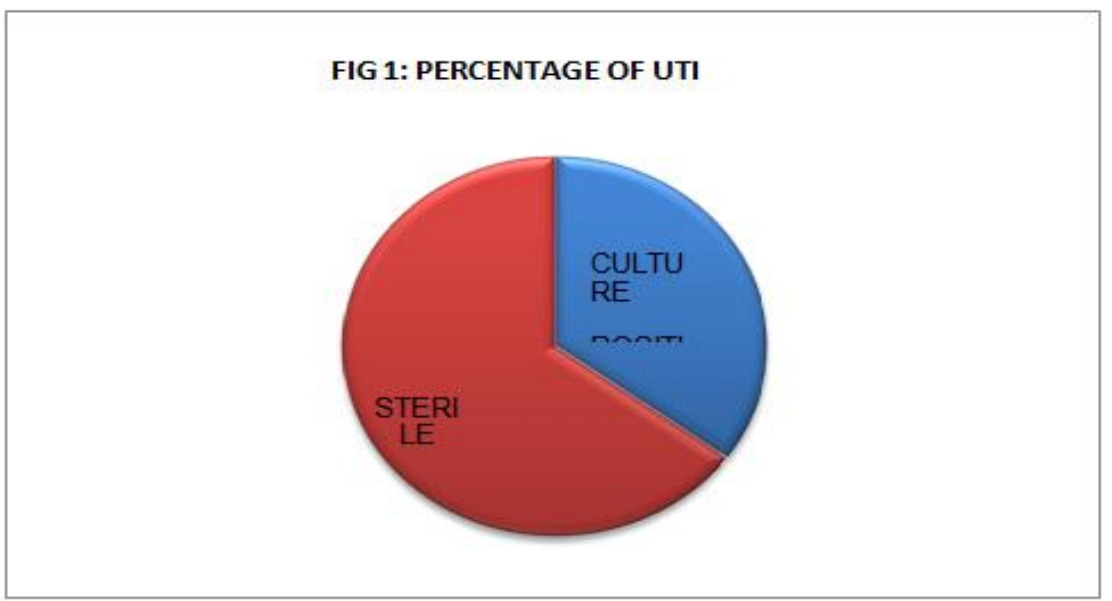

Fig.2 Ratio of Culture Positive Samples

FIG 2, RATIO OF CULTURE POSITIVE SAMPLES

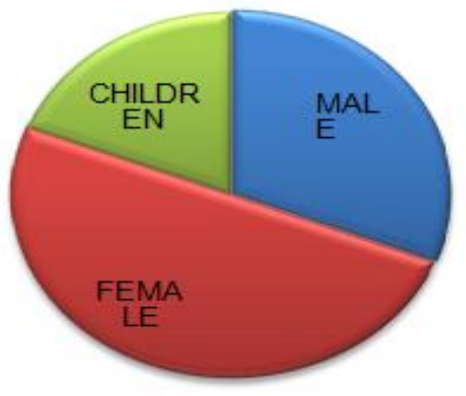

Fig.3 Isolated Pathogens with their Prevalence Rate

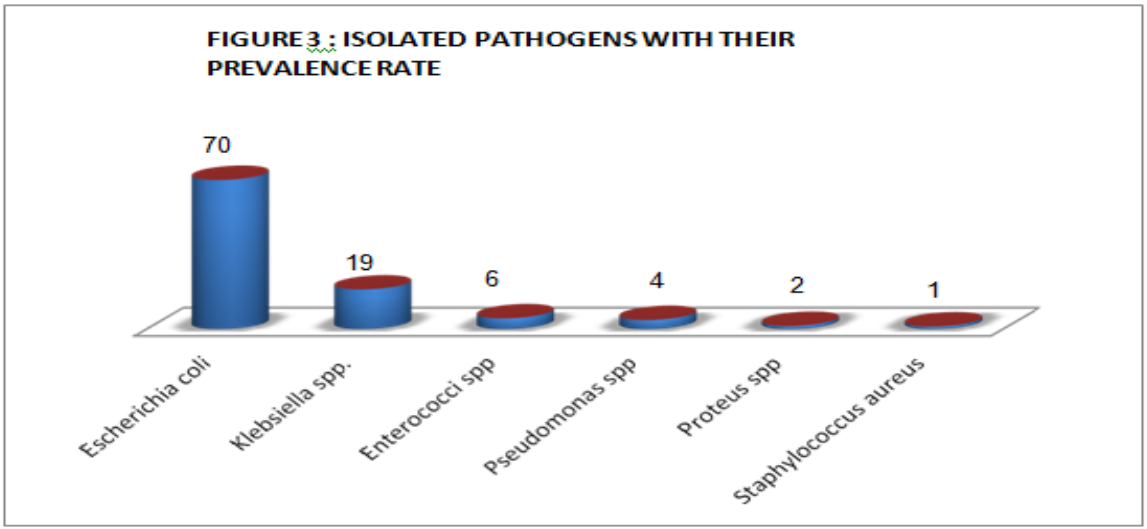


Fig.4 Antibiotic Susceptibility Pattern of E.Coli (UPEC)

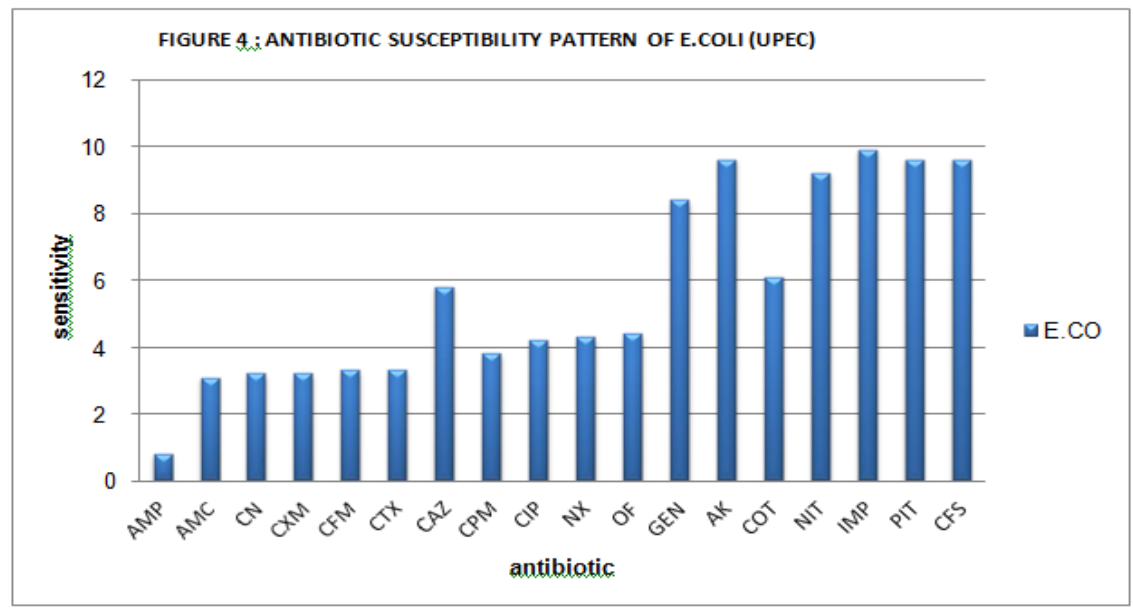

The prevalence of UTI in the study is 35\% which is similar Patel et al., (36.48\%). In comparison to our result; (Pratap et al., 2016) found that prevalence rate of UTI was $32.5 \%$. The findings of the is in accordance with other studies that females $(50 \%)$ have a notable frequency of UTI versus males $(31 \%)$. This difference in frequency could be due to several clinical factors including anatomic differences, hormonal effects, and behavioral patterns. In the study Uropathogenic E. coli (UPEC) is the major pathogen causing UTI in all category of patients which account for $70 \%$ followed by Klebsiella spp. 19\%, Enterococcus spp. 6\% (38), Pseudomonas spp. 4\% (32), Proteus spp. 2\% (11) and Staphylococcus aureus 1\% (9). This result is in agreement with the previous studies of (Salunke and Gidamudi, 2017; Sanjee et al., 2017; Vranic et al., 2017; Wong et al., 2017) in which similar organisms were isolated, with E.coli was the most prevalent isolate.

According to the antibiogram of the isolated UPEC, the higher rate of sensitivity were towards imipenem /meropenem (99\%), piperacillin - tazobactam (96\%), cefaperazone- sulbactam (96\%), amikacin (96\%) nitrofurantoin $(92 \%)$ and gentamicin $(84 \%)$. The most sensitive oral antibiotics were nitrofurantoin (92\%) followed by trimethoprim / sulfamethoxazole ie cotrimoxazole $(61 \%)$ and the quinolone group (43\%). Cephalosporin group of antibiotics showed a sensitivity percentage of $31-33 \%$ irrespective of their generation except for ceftazidime which showed a higher sensitivity percentage (58\%). Most of the isolates were resistant to ampicillin (92\% resistant). The study of (Maleki et al., 2017) found that the UPEC isolates were most resistant to ampicillin (82.9\%) \& most sensitive to nitrofurantoin $(95.9 \%)$, gentamicin $(77.2 \%)$, and amikacin $(71.5 \%)$.

This finding is in agreement with the study of (Thangavel et al., 2017) who reported that the higher rate of sensitivity among UPEC isolate were towards Amikacin (96.3\%), Imipenem (96.3 \%), and Nitrofurantoin(89.7\%). (Munkhdelger et al., 2017) explained the higher sensitivity rate towards nitrofurantoin due to the lower frequency of the use of this drug. The susceptibility patterns of E.coli against different antibiotics vary in different geographical regions, eventually leading to empirical therapy which is based on the local susceptibility profiles. Bacterial biofilm are often associated with long-term persistence of organism in various environments. Bacteria in biofilm display dramatically increased resistance to antibiotics (Tajbakhsh et al., 2016). 
The highest rate of resistance among UPEC is mainly due to unwise use of antibiotics. The increased accessibility of antibiotics probably an important factor in antibiotic resistance, Discontinuation of treatment due to negligence of patient or unavailability of drugs also develop antibiotic resistance (Chander, 2016).

The practice of prescribing antibiotics to treat UTI without bacterial characterization led to increased resistance among uropathogens and to decrease infectiveness of oral therapies.

Despite clinical symptoms of UTIs have been ameliorated by numerous antibiotics, UPEC persistence and resistance to antibiotics represent a serious problem (Blango and Mulvey, 2010). Therefore important measures are mandatory to ensure rational and wellinformed use of antibiotics.

The high incidence rate of UTIs reported in this study should be of great concern, as not only do UTIs pose a threat to health, but they also impose an economic and social burden. Increasing rate of resistance to commonly used antibiotics is an alarming sign for future of healthcare sector. Injudicious use of antibiotics could be the most important reason for such high rate of increasing resistance. Therefore, empirical treatment should be carefully selected based upon the susceptibility data of isolates as there is variation in antibiotic susceptibility pattern of UPEC. Antimicrobial stewardship has become a highly important measure in the struggle to preserve the effectiveness of available antimicrobials.

\section{References}

Al-Jebouri, M. M. 1989. The Effect of Sublethal Concentrations of Disinfectants on Antibiotic-Resistant Staphylococcus aureus. J. Hospital Infec, 14(4):14-19.
Bartoletti, R., Cai, T., Wagenlehner, F. M., Naber, K. and Johansen, T. E., 2016. Treatment of urinary tract infections and antibiotic stewardship. Eur. Urol Supplements.15(4):81-88.

Biedenbach, D. J., Badal, R. E., Huang M. Y., Motyl, M, Singhal, P. K., Kozlov, R. S., Roman, A. D. and Marcella, S 2016. In Vitro activity of oral antimicrobial agents against pathogens associated with community-acquired upper respiratory tract and urinary tract infections: a five country surveillance study. Infectious diseases and therapy.5(2):139-153.

Blango, M. G. and Mulvey, M. A., 2010. Persistence of uropathogenic Escherichia coli in the face of multiple antibiotics. Antimicrob Agents Chemotherapy.54(5):1855-1863.

Chander, M. P., 2016. Antibiotic Susceptibility of Uropathogenic E. coli Isolates from Hospitalized Patients in Warangal

City. Int.J.Curr.Microbiol.App.Sci.5(10):1619.

CLSI. Performance Standards for Antimicrobial Susceptibility Testing; Twenty- seventh Informational Supplement. CLSI document M100 : Clinical and Laboratory Standards Institute; 2017

Das, R. N., Chandrashekhar, T. S., Joshi, H. S., Gurung, M., Shrestha, N. and Shivananda, P. G., 2006. Frequency and susceptibility profile of pathogens causing urinary tract infections at a tertiary care hospital in western Nepal. Singapore medical journal. 47(4):p. 281.

Durgesh Dasharath Mahajan, Pragati Abhimanyu Bulle Microbiological Profile and Antibiogram of Bacterial Isolates Causing Urinary Tract Infection in Tertiary Care Hospital. IOSR Journal of Dental and Medical 
Sciences (IOSR-JDMS) e-ISSN: 22790853, p-ISSN: 2279-0861. Volume 15, Issue 9 Ver. V (September). 2016), PP 145-149 www.iosrjournals.org 5.

Haque, R., Akter, M. L. and Salam, M. A., 2015. Prevalence and susceptibility of uropathogens: A recent report from a teaching hospital in Bangladesh. BMC research notes. 8(1):416.

Hisano, M., Bruschini, H., Nicodemo, A. C., Gomes, C. M., Lucon, M. and Srougi, M., 2015. The bacterial spectrum and antimicrobial susceptibility in female recurrent urinary tract infection: how different they are from sporadic single episodes? Urology. 86(3):492-4

Humayun, T., Iqbal, A. 2012. The Culture and Sensitivity Pattern of Urinary Tract Infections in Females of Reproductive Age Group. Ann.Pak.Inst.Med.Sci., 8(1):19- 22.

Kumar, S., Budhani, D. and Sayal, P., $2016 b$. Bacterial Uropathogens and Empirical Treatment in Urinary Tract Infection in a Tertiary Care Institute. Int.J.Curr.Microbiol. App. Sci.5(4):4754.

Lavigne, J. P., Bruyère, F., Bernard, L., Combescure, C., Ronco, E., Lanotte, P., Coloby, P., Thibault, M., Cariou, G., Desplaces, N. and Costa, P. 2016. Resistance and virulence potential of uropathogenic Escherichia coli strains isolated from patients hospitalized in urology departments: a French prospective multicentre study. J. Med. Microbiol.65(6):530-537.

LekhaTuli, Sujeet Rai, Dilshad Arif, Deepak K. Singh. 2016. Bacteriological profile and Antimicrobial Susceptibility pattern of isolates from Urinary Tract Infections in Eastern Uttar Pradesh, India. Int.J.Curr.Microbiol.App.Sci. 5(3):428-435.

Maleki, D., Jahromy, S. H., Karizi, S. Z. and Eslami, P., 2017. The prevalence of
acrA and acrB genes among multipledrug resistant uropathogenic Escherichia coli isolated from patients with UTI in Milad Hospital, Tehran. Avicenna. J. Clin. Microb. Infec. 4(1).

Mangal, N., Vyas, A., Kumar, M. and Dalal, A. S., 2017. Antibiogram of Escherichia coli Isolates from Community Acquired Urinary Tract Infection: Special Reference to Fluoroquinolones Resistance. Int. J. Curr. Microbiol. App. Sci. 6(7):22-31.

Munkhdelger, Y., Gunregjav, N., Dorjpurev, A., Juniichiro, N. and Sarantuya, J., 2017. Detection of virulence genes, phylogenetic group and antibiotic resistance of uropathogenic Escherichia coli in Mongolia. The Journal of Infection in Developing Countries.11(01):51-57.

O'Brien, V. P., Hannan, T. J, Nielsen, H. V. and Hultgren, S. J., 2016. Drug and vaccine development for the treatment and prevention of urinary tract infections. Microbiology Spectrum. 4(1):1-62.

Patel S, Taviad P P, Sinha M, Javadekar T B, Chaudhari V P. Urinary tract infections (UTI) among patients at GG hospital and medical college, jamnagar. Nat JComm Med. 2012;3(1):138-41.

Polse, R. F., Yousif, S. Y. and Assafi, M. S., 2016. Prevalence and antimicrobial susceptibility patterns of uropathogenic E. coli among people in Zakho, Iraq. International Journal of Research in Medical Sciences. 4(4):1219-1223.

Pratap, R., Kumar, A. and Aslami, A. N., 2016. Prevalence and Antibiotic Susceptibility Pattern of Escherichia coli Positive Urinary Tract Infections in a Rural Tertiary Care Hospital in Rohtas, Bihar, India. Int.J.Curr.Microbiol.App.Sci.5(10): 128-134. 
Pratap, R., Kumar, A. and Aslami, A. N., 2016. Prevalence and Antibiotic Susceptibility Pattern of Escherichia coli Positive Urinary Tract Infections in a Rural Tertiary Care Hospital in Rohtas, Bihar, India. Int.J.Curr.Microbiol.App.Sci.5(10):128 $-134$.

Salunke, G. V., and Gidamudi, S.S., 2017. Antibiotic Susceptibility of bacterial strains with special reference to Escherichia coli isolated from urinary tract infections in rural Maharashtra. Asian J Pharm Clin Res.10(1):202205.

Sanjee, S. A., Karim, M. E., Akter, T., Parvez, M. A. K., Hossain, M., Jannat, B. and Pervin, S., 2017.Prevalence and Antibiogram of Bacterial Uropathogens of Urinary Tract Infections from a Tertiary Care Hospital of Bangladesh. Journal of Scientific Research. 9(3):317-328.

Shanthi, J., and Kayathri, S. 2012. Incidence, distribution and antibiogram of uropathogens isolated from patients with urinary tract infections. Adv Applied Sci Res.3:3410-3414.

Stefaniuk, E., Suchocka, U., Bosacka, K. and Hryniewicz, W., 2016. Etiology and antibiotic susceptibility of bacterial pathogens responsible for communityacquired urinary tract infections in Poland. Eur J ClinMicrobiol Infect Dis. 35(8):1363-1369.

Tajbakhsh, E., Ahmadi, P., Abedpour-
Dehkordi, E., Arbab-Soleimani, N. and Khamesipour, F., 2016. Biofilm formation, antimicrobial susceptibility, serogroups and virulence genes of uropathogenic E. coli isolated from clinical samples in Iran. Antimicrob Resist Infect Contro. 5(1):p.11.

Thangavel, S., Maniyan, G., Vijaya, S. and Venkateswaran, C., 2017. Bacteriological Profile of Uropathogens and their Antimicrobial Susceptibility Pattern in Isolates from a Tertiary Care Hospital. Int. J. Curr. Microbiol. App. Sci.6(5):2279-2286.

Vranic, S. M., Zatric, N., Rebic, V., Aljicevic, M. and Abdulzaimovic, A., 2017. The Most Frequent Isolates from Outpatients with Urinary Tract Infection. Materia sociomedica.29(1):17.

Werneburg, G. T., Henderson, N. S., Portnoy, E. B., Sarowar, S., Hultgren, S. J., Li, H. and Thanassi, D. G., 2015. The pilus usher controls protein interactions via domain masking and is functional as an oligomer. Nat. Struct Mol Biol.22(7):540-546.97.

Wong, C. K. M., Kung, K., Au-Doung, P. L. W., Ip, M., Lee, N., Fung, A. and Wong, S. Y. S., 2017. Antibiotic resistance rates and physician antibiotic prescription patterns of uncomplicated urinary tract infections in southern Chinese primary care. PloS one. 12(5): p.e0177266.

\section{How to cite this article:}

Jisha, T. U. and Sara Mathew. 2021. Microbiological Profile and Antibiotic Susceptibility Pattern of Urinary Tract Infections at Muthoot Medical Centre, Pathanamthitta, India. Int.J.Curr.Microbiol.App.Sci. 10(04): 903-913. doi: https://doi.org/10.20546/ijcmas.2021.1004.097 